\title{
EFEITOS DA EXIGÊNCIA DE DESEMPENHOS ENTRELAÇADOS SOBRE LINHA DE BASE EM ESQUEMA SIMPLES DE REFORÇO
}

\section{EFFECTS OF A DEMAND FOR INTERLOCKING PERFORMANCES OVER BASELINE IN SIMPLE SCHEDULES OF REINFORCEMENT}

\author{
THAIS FERRO NOGARA DE TOLEDO \\ UnIVERSIDADE Federal de Mato Grosso, Brasil \\ MARCELO FROTA Lobato BenVENUTI \\ UNIVERSIDADE DE SÃo PAULO, BRASIL
}

\section{RESUMO}

Foram investigados os efeitos da exigência de desempenhos entrelaçados, espaçamento de respostas de três participantes, sobre o responder de tríades em esquemas de reforço em intervalo variável. A tarefa consistiu em um jogo, no qual a única resposta possível, clicar o botão do mouse, era livre para ocorrer e podia produzir consequências individuais (em VI $3 \mathrm{~s}$ ou VI $12 \mathrm{~s}$ ) e consequências contingentes à exigência de que os participantes clicassem espaçadamente em relação uns aos outros. Doze estudantes universitários formaram quatro tríades. Duas condições foram programadas em um delineamento de reversão $\mathrm{ABAB}$ : Condição $\mathrm{A}$, apenas contingência operante; Condição $\mathrm{B}$, contingências operante e cultural em vigor. Os resultados mostraram que, independente do parâmetro do esquema de reforçamento individual, a sobreposição da exigência de desempenho entrelaçado selecionou um padrão de coordenação entre as respostas dos participantes. A suspensão e reintrodução da exigência do entrelaçamento mostrou que mudanças sistemáticas no padrão de ocorrência dos desempenhos entrelaçados foram função da variável manipulada. A exigência de espaçamento entre as respostas dos participantes para produção de uma consequência arranjada experimentalmente pode ser caracterizada como uma metacontingência. $\mathrm{O}$ procedimento, nesse sentido, demonstra a atuação de níveis diferentes de variação e seleção em ação simultaneamente para o comportamento dos participantes e para desempenhos entrelaçados.

Palavras-chave: Seleção cultural, contingências comportamentais entrelaçadas, esquemas de reforço, operante livre.

\section{ABSTRACT}

This study investigated the effects of an interlocking performance requirement, spacing of responses of three participants, on the triads' operant pattern in variable interval schedule of reinforcement. The task consisted of a kind of game in which the only possible response, click the mouse button, was free to occur and could produce individual consequences (in VI $3 \mathrm{~s}$ or VI $12 \mathrm{~s}$ ) and consequences contingent on the requirement that participants to click spaced with respect to each other. Twelve college students constituted four triads. Two conditions were programmed in a reversal design $\mathrm{ABAB}$ : Condition $\mathrm{A}$, only operant contingency was in effect; Condition $\mathrm{B}$, operant and cultural contingencies in effect. The results showed that, regardless of the individual schedule of reinforcement parameters, the overlap of an interlocking performance requirement selected a pattern of coordination among participants' responses. The suspension and reintroduction of interlocking requirement showed that systematic changes in the pattern of occurrence of interlocking behavior were function of the manipulated variable. The requirement of spacing between participants' responses to produce an experimentally arranged consequence can be characterized as a metacontingency. The procedure, in this sense, demonstrates the effects of different levels of variation and selection in action simultaneously for individual behavior and for interlocking performances.

Key words: Cultural Selection, interlocking behavioral contingencies, schedules of reinforcement, free operant.

\footnotetext{
Preparação deste manuscrito contou com apoio da CAPES e da Fundação de Amparo à Pesquisa do Estado de Mato Grosso (FAPEMAT, processo\#392421/2012), bem como do CNPq (processo\#573972/2008-7) e FAPESP (processo\#08/57705-8), ambos para o Instituto Nacional de Ciência, Tecnologia sobre Comportamento, Cognição e Ensino (INCT ECCE) coordenado pela Dra. Deisy G. de Souza (UFSCar). Correspondência relativa a este artigo deve ser enviada a: thaisnogara@hotmail.com ou marcelobenva@gmail.com
} 
No comportamento social, a resposta de um indivíduo está sob controle de estímulos discriminativos e/ou produz reforçadores que são mudanças no comportamento de outro (Skinner, 1953). Ao lidar com duas ou mais pessoas interagindo, deve ser levado em conta o fato de que o comportamento de um é ação que altera o mundo ao redor e também ambiente no qual o comportamento da outra pessoa (ou outras) acontece. As contingências operantes que descrevem o comportamento dessas pessoas em interação podem ser chamadas de contingências comportamentais entrelaçadas (CCEs) (ver, por exemplo, Glenn, 1991).

A demonstração experimental de que o comportamento de um indivíduo é parte relevante do ambiente em que outro se comporta é desafiadora do ponto de vista metodológico e passível de gerar "falsos positivos". Para o estudo da cooperação, por exemplo, Cohen e Lindsley (1964) desenvolveram um aparato experimental em que, em cômodos separados, dois participantes sentavam-se em frente a um painel contendo um êmbolo. Uma resposta cooperativa era registrada quando as respostas de puxar os êmbolos dos dois participantes ocorressem em um intervalo de tempo de até $0,5 \mathrm{~s}$ uma da outra. A resposta de um participante era indicada para o seu parceiro por meio de uma luz. No entanto, Schmitt e Marwell (1968) mostraram que, no procedimento de Cohen e Lindsley, altas taxas de cooperação ocorriam mesmo quando o comportamento de um participante não estava sob o controle do comportamento do outro. Os autores propuseram uma alteração na exigência para o reforçamento, adicionando uma exigência de atraso entre as respostas dos participantes. $\mathrm{Na}$ tarefa modificada, era preciso um intervalo maior do que $3 \mathrm{~s}$ e menor do que 3,5 s entre as respostas dos dois participantes. A resposta do primeiro participante acendia a luz de resposta no painel do outro participante por um período de $3 \mathrm{~s}$, o reforço ocorria se o segundo participante puxasse o êmbolo em até 0,5 s após a luz ter sido apagada. Tal alteração se mostrou efetiva em garantir que os participantes estivessem respondendo sob o controle dos comportamentos uns dos outros.

Estudos como os de Cohen e Lindsley (1964) e Schmitt e Marwell (1968) estavam interessados em explicar o comportamento individual. Suas análises se restringiam ao nível operante, ontogenético. Ao trabalharem com suas respectivas linhas de investigação, mostraram que a exigência de espaçamento entre respostas de pelo menos dois indivíduos pode ser utilizado como uma medida de relacionamento de um indivíduo com outro. O registro desse espaçamento, quando acontece, mostra a ocorrência de entrelaçamento de comportamentos (CCE). Essa estratégia pode ser útil para a investigação experimental de situações nas quais pessoas trabalham em grupo, e, especialmente, quando é possível caracterizar o grupo como um todo organizado, como uma unidade em si mesmo. Conceitualmente, a proposição do conceito de metacontingência representou um passo importante na discussão dessa possibilidade a partir das contribuições da análise do comportamento. Metacontingências podem ser definidas como "contingências comportamentais entrelaçadas que produzem um efeito agregado sobre o qual a ação de um ambiente externo é contingente" (Vichi, Andery, \& Glenn, 2009, p. 42). Por ser contingente aos comportamentos entrelaçados de duas ou mais pessoas, a ação do ambiente externo pode ser chamada consequência cultural. A consequência cultural seria responsável pela seleção e manutenção de CCEs e seus efeitos. Assim, metacontingências seriam as contingências de seleção cultural (Glenn, 2004).

Vichi et al. (2009) realizaram o primeiro estudo experimental sobre seleção cultural empregando as noções de CCEs e metacontingência. $\mathrm{O}$ procedimento consistiu em apresentar uma consequência comum para o grupo contingente a um determinado tipo de distribuição de ganhos. Grupos de quatro participantes escolhiam, consensualmente e a cada tentativa, uma linha em uma matriz 8x8. A intersecção entre linhas e colunas poderia gerar acerto ou erro (ganho ou perda de fichas trocáveis por dinheiro), o que dependia de como os participantes haviam distribuído entre si os ganhos obtidos na tentativa anterior. Duas condições foram planejadas em um delineamento de reversão: uma condição requeria distribuição igualitária dos ganhos para que uma consequência cultural fosse liberada e a outra condição exigia distribuição desigual. Aqui, as decisões sobre a divisão de ganhos, resultando em um produto (distribuição igual ou desigual) constituíam as CCEs às quais uma dada consequência (consequência cultural) era contingente. Os grupos atingiram o critério de estabilidade em cada condição experimental, ou seja, padrões distintos de distribuição dos ganhos (igual ou desigual) foram selecionados por uma exigência do experimentador de certa coordenação dos comportamentos dos participantes. A recorrência de um padrão particular de distribuição de ganhos (produto do entrelaçamento dos comportamentos de participantes) em função de uma dada consequência é o que caracteriza a seleção cultural. A relação funcional entre CCEs (e seus produtos) e a consequência selecionadora, é o que chamamos de metacontingência.

Desde o trabalho de Vichi et al. (2009), outros estudos buscaram criar tarefas experimentais e desenvolver procedimentos para o estudo experimental de metacontingências (e.g. Amorim, 2010; Baia, Azevedo, Segantini, Macedo, \& Vasconcelos, 2015; Borba, da Silva, Cabral, de Souza, Leite, \& Tourinho, 2014; Cavalcanti, Leite, \& Tourinho, 2014; Costa, Nogueira, \& Vasconcelos, 2012; Marques \& Tourinho, 2015; Morford \& Cihon, 2013; Ortu, Becker, Woelz, \& Glenn, 2012; Pavanelli, Leite, \& Tourinho, 2014; Vichi, 2012; Saconatto \& Andery, 2013; Sampaio et al., 2013; Smith, Houmanfar \& Louis, 2011; Todorov \& Vasconcelos, 2015). Além de contribuírem para o avanço das discussões sobre práticas e fenômenos culturais, esses estudos oferecem suporte empírico à noção de metacontingência como uma unidade de análise de seleção cultural.

No que se refere à seleção ontogenética, o estudo do efeito de contingências R-S em procedimentos de 
operante livre contribuiu para afirmar e debater a extensão da noção de comportamento operante e o papel da consequência para a determinação do comportamento (Skinner, 1938/1961). A estratégia de investigação usando o operante livre tornou possível a demonstração dos padrões característicos dos esquemas de reforço, produzidos pela exigência de critérios adicionados à relação "se-então" das contingências R-S (Ferster \& Skinner, 1957). O estudo do comportamento em procedimentos de operante livre foi o passo inicial para a análise experimental do comportamento e também foi importante para o desenvolvimento de pesquisas sobre o papel dos intervalos entre respostas como aspecto determinante dos padrões gerados em contingências R-S (Zeiler, 1977); as condições para o estabelecimento de controle de estímulos (e.g., Terrace, 1966); farmacologia comportamental (e.g., Dews, 1978); comportamento de escolha e preferência (e.g., Herrnstein, 1961, 1971); esquiva (e.g., Sidman, 1953); ansiedade (e.g., Estes \& Skinner, 1941), entre outras.

No contexto da discussão sobre a noção de metacontingências, Todorov e Vasconcelos (2015) e Toledo et al. (2015), realizaram experimentos para a análise da interação de duas ou três pessoas em tarefas com características similares aos procedimentos de operante livre. No procedimento de Toledo et al., a resposta requerida (pressionar o botão do mouse) era livre para ocorrer e o experimentador podia planejar diferentes critérios para a apresentação de consequências para o comportamento operante de cada participante. Simultaneamente, a tarefa permitia também que fossem programadas consequências contingentes a CCEs, definidas como respostas de cada um dos participantes espaçadas pelo tempo definido pelo experimentador. Na tarefa criada por Toledo et al., consequências individuais podiam ser programadas de acordo com um dado esquema de reforço (intervalo variável - VI, intervalo fixo - FI, razão variável - VR, razão fixa - FR, reforço contínuo - CRF). A apresentação de consequências para desempenhos entrelaçados, por outro lado, dependia da coordenação temporal entre as respostas dos participantes. A novidade nessa maneira de investigar questões levantadas pela noção de metacontingência é a possibilidade do planejamento simultâneo de níveis de variação e seleção definidos por consequências diferentes, que podem ser sobrepostas: um nível envolvendo o comportamento individual; outro, uma prática do grupo.

A presente proposta utiliza a tarefa criada por Toledo et al. (2015) para analisar interação entre desempenho individual e desempenho entrelaçado, dada a sobreposição de consequências contingentes ao entrelaçamento dos comportamentos de três participantes à consequências contingentes aos comportamentos individuais. O objetivo do estudo foi investigar os efeitos da sobreposição de uma exigência para desempenho entrelaçado sobre o desempenho operante em esquema de reforço em intervalo variável (VI 3s e VI 12s).

\section{MÉTODO}

\section{Participantes}

Participaram do experimento 12 estudantes universitários (3 do sexo feminino e 9 do sexo masculino, com idades entre 19 e 23 anos). O recrutamento foi realizado por meio de contato pessoal dos pesquisadores. Uma carta de esclarecimentos e consentimento foi utilizada no recrutamento dos participantes. O projeto foi aprovado pelos comitês de ética em pesquisa do Instituto de Psicologia da Universidade de São Paulo (471.795) e da Universidade Federal de Mato Grosso (488.187).

\section{Equipamentos e Setting experimental}

O software Culturante Livre (Toledo et al., 2015), foi utilizado para: (1) controlar a apresentação da tarefa, (2) arranjar as contingências das condições experimentais, e (3) registrar os dados do experimento.

As sessões experimentais ocorreram em uma sala de aproximadamente $20 \mathrm{~m}^{2}$. Três computadores-clientes (com mouse e sem teclado) foram dispostos lado a lado, em mesas adjacentes. O computador servidor ficava em outra mesa, afastada cerca de $1 \mathrm{~m}$ das demais.

\section{Procedimento}

Os 12 participantes foram distribuídos em quatro tríades. Quando cada participante estava sentado diante de seu respectivo computador, o experimentador ativava o programa e lia as seguintes instruções impressas em uma folha:

"A atividade que vocês irão realizar consiste num jogo, por meio do qual vocês poderão acumular dinheiro. Vocês devem descobrir a melhor maneira de produzir ganhos. Neste jogo, a função de vocês é ajudar moradores de uma pequena cidade, que estão há 5 dias sem água. Vocês podem contribuir enchendo galões de água. $\mathrm{Na}$ tela do computador existem três quadrantes, cada um designado a um jogador. Cada quadrante aparece com uma cor de fundo para o seu respectivo jogador (verde para o participante da esquerda, cor-derosa para o participante do meio e amarelo para o participante da direita). Em cada quadrante, há uma torneira e um galão (abaixo da torneira), você pode encher galões pressionando o botão do mouse sobre a sua respectiva torneira (não adianta clicar nas torneiras dos outros participantes ou em qualquer outra parte da tela). Cada vez que um participante clica sobre a sua torneira, o seu quadrante pisca para os demais. Sua ajuda será recompensada com uma quantia em dinheiro. Cada galão completo lhe renderá $\mathrm{R} \$ 0,01$. Acima dos quadrantes, há um espaço que representa uma área comum, onde há uma caixa d'água. Em alguns períodos, será possível produzir um bônus que faz com que a caixa d'água da cidade seja preenchida. Para isso, o grupo terá que trabalhar em conjunto e tentar descobrir qual é a estratégia que produz bônus. Os bônus produzidos ficam registrados no hidrômetro, ao lado da caixa d'água. Cada bônus acumulado renderá ao grupo $\mathrm{R} \$ 0,05$. Tentem produzir a maior quantidade de água que conseguirem. Vocês 
podem conversar sobre a tarefa o quanto quiserem, fiquem à vontade. Bom trabalho!"

Durante todo o experimento, as folhas contendo as instruções impressas ficavam disponíveis ao lado dos computadores dos participantes.

Tarefa Experimental e Contingências

\section{Programadas.}

$\mathrm{Na}$ tela dos computadores-clientes apareciam 3 quadrantes, um para cada participante, com uma torneira e um galão (ver Figura 1). Acima dos quadrantes havia uma área comum, na qual havia uma caixa d'água. Cada participante via o seu quadrante com uma cor de fundo diferente (verde para o participante da esquerda, rosa para o do meio e amarelo para o da direita). O quadrante colorido era o único ativo para aquele participante, os outros dois quadrantes apareciam com um fundo branco. Sempre que um participante emitia uma resposta, seu quadrante piscava na tela dos outros participantes (aproximadamente $0,2 \mathrm{~s}$ ). Isso servia para indicar para cada participante quando os outros estavam respondendo. A Figura 1 ilustra a tela do computador de um participante na posição da esquerda (verde). À esquerda, mostra o exato momento em que o participante cujo quadrante é amarelo emite uma resposta.

A tarefa dos participantes consistia em clicar com o botão esquerdo do mouse sobre a torneira que aparecia em seu respectivo quadrante. Os eventos programados para ocorrer após essa resposta eram de dois tipos: porções de água, que enchiam o galão (os galões acumulados ficavam registrados no ícone de galão em miniatura, localizado em cada quadrante) e bônus, que eram indicados pela palavra "bônus" na tela e o acréscimo de porções de água na caixa de água comum. A quantidade de bônus obtida a cada sessão ficava registrada no ícone de um hidrômetro, localizado na área comum, acima dos quadrantes dos participantes (ver Figura 1, à direita). Tanto os galões, quanto os bônus acumulados eram posteriormente trocados por uma quantia em dinheiro. Cada galão rendia $\mathrm{R} \$ 0,01$ ao participante e cada bônus rendia $\mathrm{R} \$ 0,05$ ao grupo.
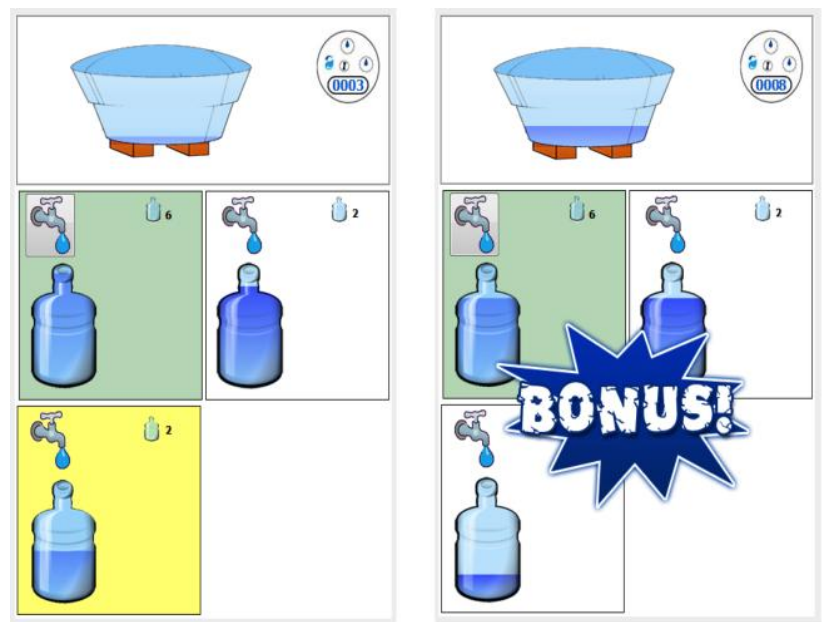

Figura 1. Tela do computador do P1 (com fundo verde) em dois momentos: no momento em que P3 clica sobre sua torneira (esquerda) e no momento em que um bônus é apresentado (direita).
Contingência para comportamento individual.

Respostas de pressionar o botão da torneira, com o mouse, produziam uma porção de água (que saía da torneira e caía no galão situado imediatamente abaixo da mesma) em um esquema de intervalo variável (VI). O reforço ficava disponível para as respostas de pressionar esse botão em média a cada 3 ou 12 segundos. Para as tríades 1 e 2 esteve em vigor um VI 3. Para as tríades 3 e 4 esteve em vigor um VI 12. Foram utilizados seis intervalos, gerados por meio de sorteio aleatório (Costa \& Banaco, 2002) para cada VI (2, 5, 4, 2, 4, 5s no VI 3; 3, 13, 10, 17, 18, 13s no VI 12). Dez porções de água enchiam um galão, o que resultava na apresentação de um som (Som 1) e a adição de 01 galão no visor situado no canto superior direito do quadrante. Neste momento, um novo galão vazio aparecia abaixo da torneira ${ }^{1}$.

O esquema de intervalo variável foi escolhido por duas razões: a primeira é que a contingência para o comportamento individual não deveria interferir ou concorrer com a exigência para o desempenho entrelaçado (como ocorre na maioria dos estudos experimentais sobre metacontingências, e.g., Amorin, 2010; Caldas, 2009; Costa et al., 2012; Morford \& Cihon, 2013; Vichi, 2012). A segunda razão é que o esquema não deveria favorecer a ocorrência de pausas, o que dificultaria a análise posterior do espaçamento entre as respostas dos participantes.

\section{Contingência para desempenho entrelaçado.}

Com essa programação, uma consequência nova poderia ser produzida quando o intervalo entre as respostas dos três participantes era maior do que $x$ milissegundos (o que variou entre $750 \mathrm{~ms}$ e $1250 \mathrm{~ms}$ no presente estudo). Quando o desempenho entrelaçado cumpria essa exigência programada pelo experimentador, a palavra "Bônus" aparecia acompanhada de um som específico. Adicionalmente, uma porção de água era adicionada à caixa d'água. Qualquer um dos participantes poderia ser o primeiro a responder na sequência que definia o entrelaçamento, mas para que o bônus fosse apresentado, o segundo participante a clicar tinha que esperar, pelo menos, $x \mathrm{~s}$ e o terceiro, mais $x \mathrm{~s}$ em relação ao segundo. O elemento chave aqui, como sugerido por Schmitt e Marwell (1968), foi a adição da exigência de um intervalo de tempo entre as respostas dos participantes, com o objetivo de tentar garantir que a resposta de um estivesse sob o controle do comportamento do outro. Uma sequência coordenada de três respostas (uma de cada participante), espaçadas de acordo com o exigido, caracterizava uma CCE. Quando se completava uma CCE, o bônus era apresentado de forma contígua à resposta do último participante. Inicialmente, o software foi programado para registrar todas as ocorrências de CCEs com espaçamento entre respostas maior ou igual a $750 \mathrm{~ms}$, mesmo quando a exigência para entrelaçamento não estava em vigor. No entanto, ao longo do experimento, esse intervalo foi alterado, aumentado ou reduzido.

\footnotetext{
${ }^{1} \mathrm{O}$ esquema utilizado pode ser caracterizado como um tipo de esquema de segunda ordem, no qual o reforço era apresentado a cada 10 cumprimentos da exigência do esquema VI.
} 


\section{Delineamento Experimental}

Duas condições foram conduzidas em um delineamento de reversão $\mathrm{ABAB}$. Na Condição $\mathrm{A}$, apenas a contingência para o comportamento individual estava em vigor; na Condição $\mathrm{B}$, as exigências para o comportamento individual e para o entrelaçamento dos comportamentos dos participantes estavam em vigor simultaneamente.

Durante a Condição A, foram registradas todas as ocorrências de eventuais entrelaçamentos nos quais as respostas dos três participantes distassem, pelo menos, 750 $\mathrm{ms}$ entre si. Isso por que um dos objetivos desta condição era fornecer uma linha de base para avaliar os efeitos, na Condição B, da exigência de desempenho entrelaçado (a variável independente do estudo) sobre a ocorrência das CCEs requeridas (a variável dependente).

O critério para o encerramento da primeira exposição à Condição $\mathrm{A}$ foi de pelo menos nove minutos, com estabilidade na ocorrência de eventuais CCEs nos três últimos minutos. Quando havia até cinco ocorrências por minuto, aceitava-se uma variação de até duas ocorrências; quando havia de seis a nove ocorrências, aceitava-se uma variação de até três ocorrências; e quando havia mais que 10 ocorrências por minuto, aceitava-se uma variação de até quatro por minuto. A segunda exposição foi encerrada quando a CCE selecionada na Condição B anterior ocorreu com uma frequência semelhante à apresentada durante os três minutos finais da primeira exposição (linha de base), por três minutos consecutivos.

Os critérios para o encerramento da Condição B foram os seguintes: (a) 12 min consecutivos com emissão de CCEs que atendiam o critério para a produção de bônus (CCE-alvo); (b) estabilidade na frequência dessas CCEs nos últimos 6 min.

A Tabela 1 apresenta o delineamento experimental, com a especificação de quais contingências estavam programadas para o desempenho individual e para e desempenho entrelaçado.

Tabela 1

Delineamento Experimental.

\begin{tabular}{|c|c|c|c|}
\hline Participantes & Condição & $\begin{array}{l}\text { Esquema de } \\
\text { reforço }\end{array}$ & $\begin{array}{l}\text { Exigência de desempenho } \\
\text { entrelaçado }\end{array}$ \\
\hline
\end{tabular}

A

$\mathrm{P} 1, \mathrm{P} 2, \mathrm{P} 3$

$\frac{\mathrm{B}}{\mathrm{A}}$

\begin{tabular}{cc} 
Clicar produz \\
\cline { 2 - 2 } $\begin{array}{c}\text { reforço em VI } \\
(3 \text { s ou 12 s })\end{array}$
\end{tabular}

*O intervalo de $x$ s foi definido ao longo do experimento.

\section{Definição do espaçamento exigido entre as respostas dos participantes na Condição B. \\ $\mathrm{O}$ intervalo entre respostas requerido para} apresentação da consequência contingente ao desempenho entrelaçado variou ao longo do experimento, a depender do desempenho de cada tríade. Durante a primeira Condição A (linha de base), CCEs com espaçamento maior ou igual a $750 \mathrm{~ms}$ eram registradas (o experimentador tinha acesso a essa informação instantaneamente, pela tela do computador servidor). Esse registro orientou a decisão do experimentador sobre o intervalo a ser empregado quando a exigência para desempenho entrelaçado entrasse em vigor. Caso fossem registradas ocorrências de CCEs com $750 \mathrm{~ms}$ de espaçamento por, pelo menos, 3 minutos consecutivos durante a linha de base, o experimentador solicitava que o programa registrasse apenas aquelas CCEs com espaçamento maior ou igual a $1000 \mathrm{~ms}$. Caso ainda houvesse muitas ocorrências destas, outros $250 \mathrm{~ms}$ eram adicionados para o critério. Isso era feito até chegar a uma CCE com baixa probabilidade de ocorrência ao acaso. $\mathrm{Na}$ primeira exposição à Condição $\mathrm{B}$, a consequência contingente ao desempenho entrelaçado (bônus) era contingente a este espaçamento. Quando acontecia o espaçamento definido de maneira estável por seis minutos consecutivos, o espaçamento exigido aumentava em 250 ms. Caso o aumento no intervalo do espaçamento exigido produzisse a ocorrência de três minutos consecutivos com uma taxa de CCE semelhante à apresentada nos três minutos finais da linha de base, o valor do espaçamento anterior era novamente implementado e este valor passava a ser o espaçamento alvo da seleção. O objetivo era estabelecer um entrelaçamento que tivesse sido pouco frequente durante a linha de base, mas que fosse passível de ser mantido a uma taxa relativamente alta e estável.

As sessões experimentais tinham duração de aproximadamente 50 minutos e foram realizadas em dias diferentes. Ao final de cada sessão, o experimentador interrompia a atividade, anotava os ganhos de cada participante e os ganhos do trio. No final do experimento, o experimentador apresentava o valor total acumulado por meio de galões (individual) e por meio de bônus e cada tríade decidia como queria dividir o valor acumulado a partir dos bônus. Os participantes tinham 3 minutos para tomar essa decisão. Em seguida, o experimentador entregava ao grupo uma folha com a seguinte pergunta: Que estratégia seu grupo utilizou para produzir bônus? Eles tinham 5 minutos para responder por escrito. 


\section{RESULTADOS}

A variável manipulada no presente estudo foi a exigência de um espaçamento entre as respostas dos participantes de uma tríade (CCEs) para a apresentação de uma dada consequência para o desempenho entrelaçado. Avaliou-se os efeitos da introdução e remoção dessa exigência sobre a ocorrência de respostas de cada um dos integrantes da tríade e sobre a ocorrência da CCE alvo, ao longo do tempo e das mudanças de condições.
Foram realizadas duas sessões com as Tríades 1, 2 e 4. Com a Tríade 3 , foram realizadas 3 sessões. Para as Tríades 1 e 3, o espaçamento final, alvo da seleção (CCE alvo), foi de $750 \mathrm{~ms}$ e para as Tríades 2 e 4, de $1000 \mathrm{~ms}$. Esses valores foram definidos pelos resultados obtidos durante a linha de base. Para a análise, são essas as CCEs que foram plotadas durante as Condições A.

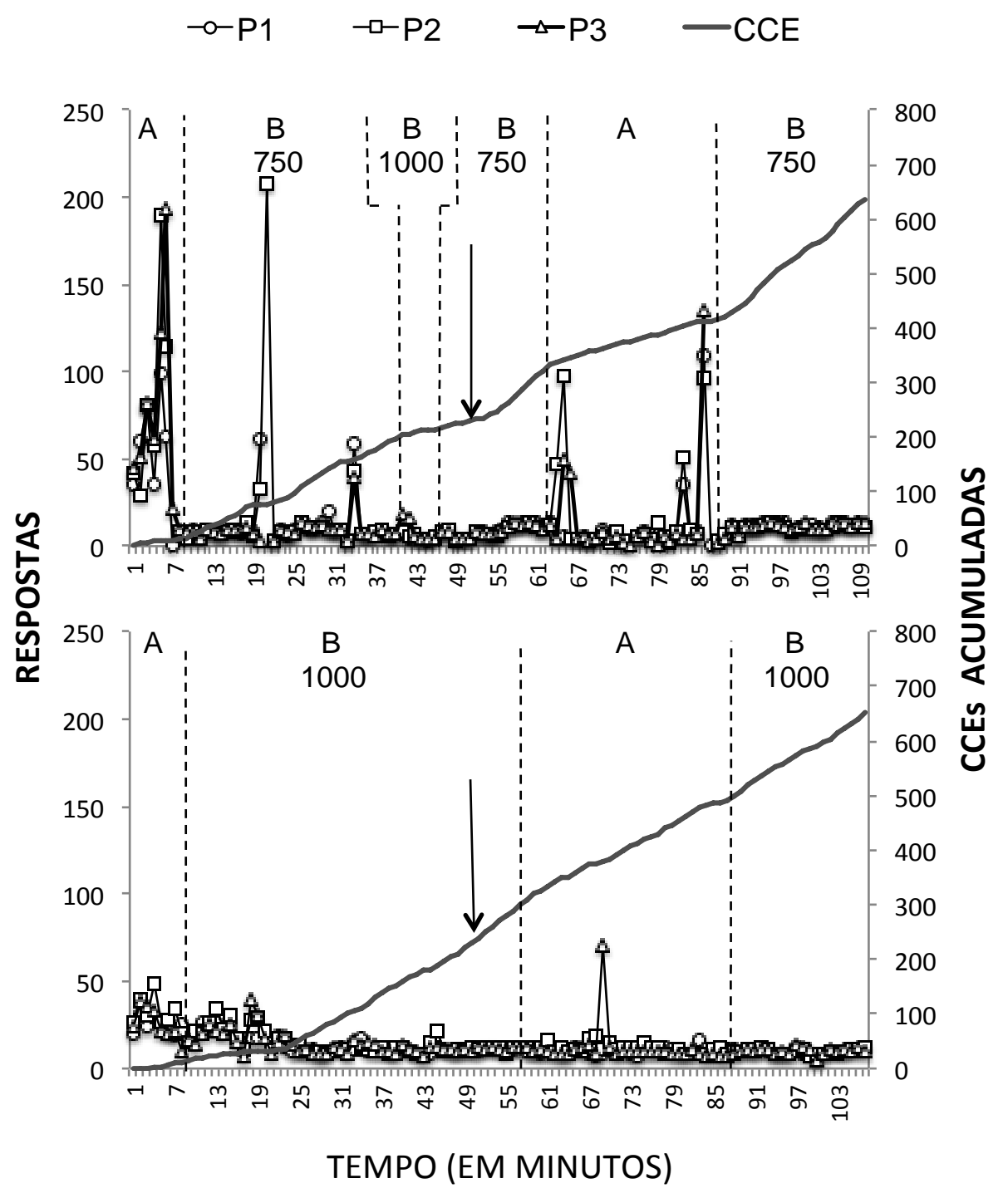

Figura 2. Número de Respostas (eixo principal) e número acumulado de CCEs (eixo secundário), por minuto, apresentados pelos participant es das Tríades 1 (painel superior) e 2 (painel inferior), em cada uma das condições experimentais. Durante as Condições A, foram plotadas as eventuais CCEs com espaçamento maior ou igual a $750 \mathrm{~ms}$ para a Tríade 1 e de 1000 ms para a Tríade 2 . As linhas tracejadas indicam mudança de condição. As setas indicam o momento em que a sessão foi encerrada para ser reiniciada em outro dia.

A Figura 2 apresenta o número de respostas por minuto e a frequência acumulada de CCEs que atingiram o critério para a produção do bônus em cada uma das condições experimentais para as Tríades 1 (painel superior) e 2 (painel inferior). Para ambas as tríades, vigorava um esquema VI $3 \mathrm{~s}$ na contingência individual. Essas duas tríades apresentaram padrões de respostas bastante diferentes desde a primeira exposição à Condição A (linha de base). Os participantes da Tríade 1 emitiram entre 35 e 190 por min durante os seis primeiros minutos da primeira exposição à condição A (linha de base). O entrelaçamento que produziria a consequência cultural, caso a exigência de espaçamento de $750 \mathrm{~ms}$ estivesse em vigor (CCE alvo), foi registrado 10 vezes durante este período (média de 1,7 por min). Nos três minutos finais, a taxa de 
ocorrência da CCE alvo manteve-se inalterada, enquanto a taxa média de respostas de cada participante passou de 80 para 8 respostas por minuto. A taxa de CCEs aumentou de 2 para 6 ocorrências logo no primeiro minuto com a exigência de entrelaçamento. Essa número de ocorrências por minuto variou entre zero e 13 ao longo da primeira Condição B (com exigência de $750 \mathrm{~ms}$ para espaçamento), tornando-se estável com 6 a 7 ocorrências por minuto no $40^{\circ}$. minuto do experimento, quando o espaçamento exigido aumentou de 750 para $1000 \mathrm{~ms}$. O aumento na exigência do espaçamento resultou em uma redução na frequência de emissão da CCE de 7 para no máximo duas ocorrências por minuto, por quatro minutos consecutivos, embora a taxa de respostas dos três participantes permanecesse baixa. Devido a essa redução abrupta na taxa de CCEs, o espaçamento de $750 \mathrm{~ms}$ foi retomado mas, apenas na segunda sessão as CCEs voltaram a ser apresentadas com uma frequência maior, atingindo o critério de estabilidade entre 10 e 12 ocorrências por minuto no $12^{\circ}$. minuto da sessão. A suspensão da consequência para desempenhos entrelaçados (segunda Condição A) resultou em maior variação nas taxas de respostas dos três participantes e redução na frequência de emissão das CCEs alvo. Após 21 minutos com variação entre 0 e 7 ocorrências por minuto, a taxa da CCE alvo retornou ao nível de linha de base (média de 1,3 por minuto), por três minutos consecutivos. A Condição B foi reintroduzida e, a partir do $5^{\circ}$ minuto, a CCE alvo voltou a ser apresentada a uma taxa próxima à obtida na Condição $\mathrm{B}$ anterior (10 a 12 por minuto).

Para a Tríade 2, as taxas de respostas dos participantes foram relativamente mais baixas, comparadas com o que foi observado para a Tríade 1. Em relação à taxa de ocorrência de CCEs, com uma exigência de espaçamento de $750 \mathrm{~ms}$, foram registradas 39 ocorrências da CCE já durante a linha de base. Com o espaçamento de $1000 \mathrm{~ms}$, foram registradas 16 ocorrências (entre 2 e 4 por minuto). Em função desse dado, quando a Condição $\mathrm{B}$ foi introduzida, o espaçamento requerido foi o de 1000 ms e não o de 750, programado anteriormente. A taxa de ocorrência da CCE alvo variou entre zero e 4 ocorrências por minuto ao longo dos primeiros 23 minutos do experimento. A partir do $24^{\circ}$ minuto, as CCEs passaram a ser emitidas com uma frequência maior, em torno de 7 ocorrências por minuto, e o critério para mudança de condição foi atingido no sexto minuto da segunda sessão. Com a suspensão da exigência para desempenho entrelaçado, no retorno à Condição $\mathrm{A}$, ocorreu uma redução e uma maior variação na taxa de ocorrência da CCE alvo. Ao final de 30 minutos de exposição a essa condição, a taxa de CCEs retornou aos níveis registrados em linha de base (uma média de 3 ocorrências por minuto), por três minutos consecutivos e a Condição B anterior foi reintroduzida. Do $15^{\circ}$ minuto até o final da sessão em que a Tríade encerrou o experimento, a taxa de CCEs manteve-se estável com 8 e 10 ocorrências por minuto.
A Figura 3 apresenta as taxas de respostas dos três participantes e a frequência acumulada de CCEs das Tríades 3 (painel superior) e 4 (painel inferior), para as quais vigorava o VI 12 s na contingência individual. De modo semelhante ao que ocorreu com os participantes da Tríade 1, os participantes da Tríade 3 iniciaram o experimento com uma alta taxa de respostas (em média, 55 respostas por minuto para $\mathrm{P} 1$ e $\mathrm{P} 2$, e 150 para $\mathrm{P} 3$ ), e essa taxa diminuiu nos minutos finais da linha de base para cerca de 14 respostas por minuto. Nestes minutos finais, a taxa de ocorrência da CCE que estava sendo mensurada (com espaçamento de $750 \mathrm{~ms}$ ) variou entre $2 \mathrm{e}$ 4. A introdução da exigência para desempenho entrelaçado, com diferentes exigências de espaçamento $(750,1000$ e $1250 \mathrm{~ms})$, não produziu alterações tanto nas taxas de respostas individuais quanta na taxa de ocorrência das CCEs. Ou seja, durante o início da Condição B, os participantes da Tríade 3 continuaram respondendo da mesma forma com que vinham respondendo durante os quatro últimos minutos da linha de base. Pode-se dizer, neste caso, que as CCEs estavam ocorrendo no nível do acaso e que a contingência operante explica tanto a taxa de respostas, quanto o padrão de entrelaçamento resultante. $\mathrm{O}$ padrão de respostas dos participantes só mudou durante a segunda exposição à Condição $\mathrm{B}$ com a exigência de espaçamento de $1000 \mathrm{~ms}$, quando houve aumento na taxa de respostas dos participantes e redução na taxa de CCEs (próximo a zero). Neste ponto, a exigência de espaçamento foi reduzida de 1000 para $750 \mathrm{~ms}$ e os três participantes passaram a apresentar cerca de 18 a 20 respostas por minuto. O critério de estabilidade para encerramento da Condição B, com 10 a 14 ocorrências da CCE por minuto, foi alcançado no $25^{\circ}$ minuto de exposição a esta condição (Minuto 133 do experimento para a Tríade 3). Com a suspensão da consequência cultural (CC), houve maior variação tanto nas taxas de respostas dos três participantes quanto na taxa de CCEs. A partir do terceiro minuto sem exigência para desempenhos entrelaçados, as CCEs passaram a ocorrer a uma taxa de 4,5 por minuto, chegando a 0,67 nos três minutos finais da condição. A reintrodução da exigência para desempenho entrelaçado, no retorno à Condição $\mathrm{B}$, resultou na reinstalação imediata do padrão de entrelaçamento anterior.

Para a Tríade 4, as taxas de respostas obtidas na primeira condição A mantiveram-se no início da primeira exposição à Condição $\mathrm{B}$, com uma média de 35 por minuto. Próximo ao $19^{\circ}$ minuto, o número de respostas individuais diminuiu e passou a haver maior número de CCEs. Quando a exigência para desempenho entrelaçado foi suspensa, no retorno à Condição $\mathrm{A}$, houve uma maior variação e redução no número de respostas emitido por cada participante por minuto. Com o retorno à Condição B (com exigência de espaçamento de 1000 ms) a tríade voltou a apresentar o padrão de respostas e de CCEs anteriormente observado: cada participante emitiu entre 9 e 12 respostas por minuto e a tríade produziu entre 9 a 12 bônus por minuto. 


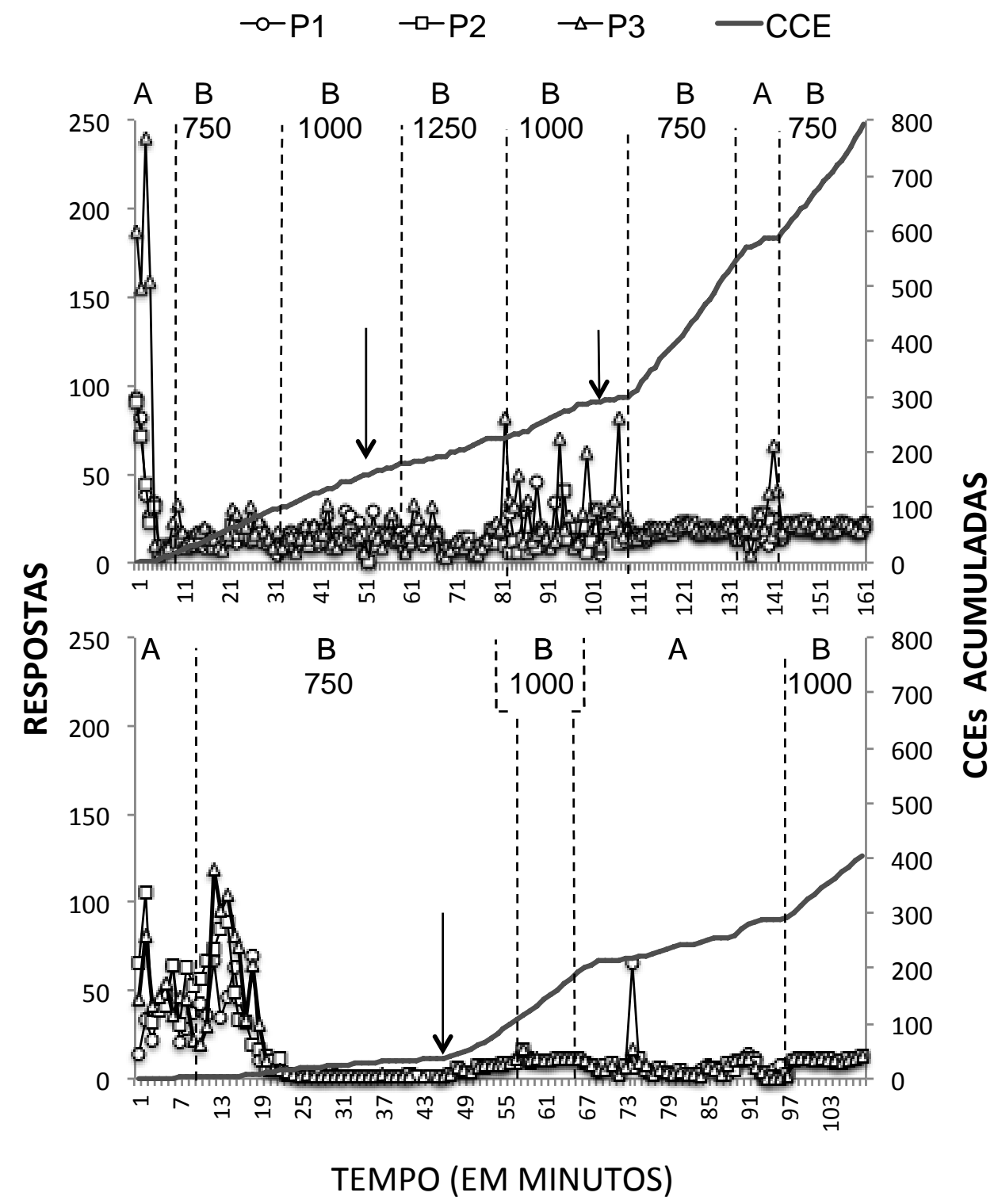

Figura 3. Número de Respostas (eixo principal) e número acumulado de CCEs (eixo secundário), por minuto, apresentados pelos participantes das Tríades 3 (painel superior) e 4 (painel inferior), em cada uma das condições experimentais. Durante as Condições A, foram plotadas as eventuais CCEs com espaçamento maior ou igual a $750 \mathrm{~ms}$ para a Tríade 3 e de $1000 \mathrm{~ms}$ para a Tríade 4 . As linhas tracejadas indicam mudança de condição. As setas indicam o momento em que a sessão foi encerrada para ser reiniciada em outro dia.

Em relação à coleta do relato verbal, os participantes das quatro tríades responderam (por escrito) que para produzir bônus os participantes tinham que clicar seguindo uma dada sequência. As Tríades 1 e 2 mencionaram ainda que deveria haver um espaço de tempo de $1 \mathrm{~s}$ (Tríade 1) e $3 \mathrm{~s}$ (Tríade 2) entre os cliques. Quanto ao dinheiro acumulado por meio dos bônus, as Tríades 1 e 4 decidiram dividir o valor obtido em três partes iguais e as Tríades 2 e 3 decidiram usar a quantia para um lanche conjunto.

\section{DISCUSSÃO}

Os resultados do presente estudo mostraram que a exigência de desempenho entrelaçado produziu um padrão de coordenação entre as respostas de participantes de tríades. Esse efeito foi observado quando essa exigência foi sobreposta a uma linha de base em que o comportamento individual estava sendo mantido por um esquema de intervalo variável. A suspensão da exigência de desempenho entrelaçado, nas quatro tríades, mostrou que a ocorrência da CCE alvo, a uma taxa relativamente alta e estável, dependeu da apresentação da consequência específica para o desempenho entrelaçado.

$\mathrm{O}$ experimento mostrou a seleção (em termos de recorrência) de uma unidade comportamental que engloba comportamentos de três indivíduos (sob controle dos comportamentos uns dos outros), por meio da apresentação de uma consequência contingente à exigência de desempenho entrelaçado. Os dados deste estudo, somados aos apresentados por outros protocolos de pesquisa (e.g., Morford \& Cihon, 2013; Ortu et al., 2012; Saconato \& Andery, 2013; Vichi et al., 2009) sugerem que 
a noção de metacontingência pode ser útil como unidade de análise de fenômenos no terceiro nível de seleção por consequências, o cultural. $\mathrm{O}$ uso de esquemas de reforço como linha de base para avaliar o efeito da sobreposição de uma exigência para o entrelaçamento dos comportamentos dos participantes pode contribuir para o avanço das investigações nesta área de pesquisa. Essa estratégia é importante porque demonstra a possibilidade de análise da relação entre desempenho individual e trabalho em grupo como produto de dois processos de seleção distintos e simultâneos.

$\mathrm{Na}$ tarefa utilizada no presente estudo, a medida do entrelaçamento (ou de CCEs) não é limitada pela duração de tentativas. Os participantes podem pressionar o botão a qualquer momento da sessão e o experimentador pode planejar diferentes critérios para a apresentação de consequências individuais e de consequências contingentes à ocorrência de desempenhos entrelaçados. Um exemplo das possibilidades de análise oferecidas pela presente estratégia de investigação é a demonstração do processo de extinção de CCEs. No presente estudo, a suspensão da consequência cultural resultou em um retorno à taxa de CCEs obtida em linha de base, resultado semelhante ao que ocorre durante a extinção do comportamento operante. Uma curva com aceleração negativa em função da remoção da exigência para desempenhos entrelaçados não tem sido obtida em experimentos com procedimentos de tentativas (ver, por exemplo, Baia et al., 2015; Borba, 2013; Caldas, 2009; Vichi, 2012). Nesses procedimentos, independente da condição em vigor, os participantes têm que emitir respostas a cada tentativa. Caso contrário, o experimento não avança. Assim, mesmo quando a consequência contingente ao entrelaçamento de comportamentos é suspensa, o grupo tem que, a cada tentativa, escolher uma linha ou inserir números ou selecionar um cartão (ou tecla), não sendo possível deixar de responder.

A presente investigação aproxima a análise sobre seleção cultural na análise do comportamento da análise que é comumente feita na área dos esquemas de reforço, permitindo uma comparação mais estreita do desempenho individual e do obtido com o entrelaçamento de contingências comportamentais. A estratégia de programar esquemas simples de reforço e utilizar o padrão de respostas gerado como linha de base para avaliar os efeitos de uma outra manipulação experimental é comum na análise experimental do comportamento (ver, por exemplo, Pierce \& Cheney, 2013). Ao programar uma consequência contingente ao entrelaçamento de comportamentos sobre uma contingência operante, o presente estudo possibilitou observar, simultaneamente, dois processos distintos de seleção em curso: seleção operante e seleção cultural.

Do modo como a contingência operante (em esquema VI) e a exigência para desempenhos entrelaçados foram programadas, cada participante poderia obter tanto os reforçadores quanto as consequências contingentes ao entrelaçamento programadas pora cada uma das condições em vigor. Os resultados mostraram que, independente do intervalo empregado no esquema individual, a frequência de respostas e de CCEs foram muito parecidas durante os períodos de estabilidade das Condições B finais, para todos os participantes: o número de respostas por bônus variou entre 1,0 e 1,5. Como previsto, ao atender as exigências para desempenho entrelaçado, a razão de respostas por reforço ficou em torno de uma resposta para cada reforço entre os participantes das tríades com esquema individual em VI $3 \mathrm{~s}$ e em torno de três a quatro respostas por reforço entre os participantes das tríades em VI $12 \mathrm{~s}$.

Quando a contingência operante e a metacontingência trabalham em conjunto, quando selecionam um mesmo padrão de respostas, é possível dizer que seleção comportamental e cultural são redundantes. Para Hunter (2012) tal redundância dificultaria a avaliação dos efeitos da apresentação da consequência cultural de modo separado do efeito das contingências operantes. $\mathrm{O}$ argumento é que, nesses casos, a contingência operante seria suficiente para explicar a recorrência das CCEs. No presente estudo, poder-se-ia supor que o esquema de intervalo variável teria selecionado um padrão de responder espaçado de cada um dos participantes da tríade, resultando na produção de reforços e, adicionalmente, de bônus. Assim, apenas a contingência operante explicaria tanto o comportamento individual quanto o entrelaçamento dos comportamentos dos participantes (CCEs). No entanto, os resultados do presente estudo mostraram que sem uma contingência específica para o espaçamento entre respostas de diferentes participantes este não ocorre a uma taxa relativamente alta e e estável, mesmo quando as taxas de respostas individuais são baixas e estáveis. A estratégia de programar simultaneamente um esquema de reforço para o comportamento individual e uma consequência distinta para os desempenhos entrelaçados dos participantes possibilita analisar a relação entre desempenho individual e desempenhos entrelaçados como uma questão de escolha simultânea e dinâmica, mais evidente com a programação de esquemas VI que produzem taxas mais altas de resposta. A programação realizada no presente estudo, com novos esquemas e valores de esquemas, pode contribuir com a discussão sobre conflito (Tourinho \& Vichi, 2012) ou concorrência entre contingências operantes e contingências culturais (e.g., Borba, 2013; Hunter, 2012; Morford \& Cihon, 2013).

Resumindo as principais contribuições do presente estudo, é possível afirmar que o emprego de um procedimento análogo ao operante livre para o estudo de contingências comportamentais entrelaçadas permite utilizar o desempenho em esquemas individuais como linha de base para exigência de desempenhos entrelaçados. O uso de uma única chave de respostas permite a análise, com simplicidade experimental, de interações momento a momento entre processos de seleção nos níveis individual e cultural.

\section{REFERÊNCIAS}

Amorim, V. C. (2010). Análogos experimentais de metacontingências: efeitos da intermitência da consequência cultural. Dissertação de Mestrado, 
Programa de Estudos Pós-Graduados em Psicologia Experimental: Análise do Comportamento, PUC-SP, São Paulo.

Baia, F. H., Azevedo, F. F., Segantini, S. M., Macedo, R. P., \& Vasconcelos, L. A. (2015). Effects of diferente magnitudes of individual consequences and cultural consequences on culturant's selection. Acta Comportamentalia, 23, 257-272.

Borba, A. (2013). Efeitos da exposição a macrocontingências e metacontingências na produção e manutenção de respostas de autocontrole ético. Tese de doutorado, Programa de Pós Graduação em Teoria e Pesquisa do Comportamento, Universidade Federal do Pará, Belém.

Borba, A., da Silva, B. R., Cabral, P. A. A., de Souza, L. B., Leite, F. L., \& Tourinho, E. Z. (2014). Effects of Exposure to Macrocontingencies in isolation and social situations in the production of ethical self-control. Behavior and Social Issues, 23, 5-19.

Caldas, R. A. (2009). Análogos experimentais de seleção e extinção de metacontingências. Dissertação de Mestrado, Programa de Estudos Pós-Graduados em Psicologia Experimental: Análise do Comportamento, PUC-SP, São Paulo.

Cavalcanti, D. E., Leite, F. L., \& Tourinho, E. Z. (2014). Seleção de Práticas culturais complexas: Avaliação experimental de um análogo do procedimento de aproximação sucessiva. Psicologia e Saber Social, 3, $2-21$.

Cohen, D., \& Lindsley, O. R. (1964). Catalysis of controlled leadership in cooperation by human stimulation. Journal of Child Psychological Psychiatry, 5, 119-137. doi: 10.1111/j.14697610.1964.tb02134.x

Costa, C. E., \& Banaco, R. A. (2002). ProgRef v3: Sistema computadorizado para a coleta de dados sobre programas de reforço com humanos - Recursos básicos. Revista Brasileira de Terapia Comportamental e Cognitiva, 4, 171-172.

Costa, D., Nogueira, C., \& Vasconcelos, L. (2012). Effects of communication and cultural consequences on choices combinations in INPDG with four participants. Revista Latinoamericana de Psicologia, 44, 121-131.

Dews, P. B. (1978). Origins and future of behavioral pharmacology. Life Science, 22(13-15): 1115-1121. doi: 10.1016/0024-3205(78)90080-2

Estes, W. K., \& Skinner, B. F. (1941). Some quantitative properties of anxiety. Journal of Experimental Psychology, 29, 390-400. doi: 10.1037/h0062283

Ferster, C. B., \& Skinner, B. F. (1957). Schedules of reinforcement. New York: Appleton-Century-Crofts.

Glenn, S. S. (1991). Contingencies and metacontingencies: Relations among behavioral, cultural, and biological evolution. In P. A. Lamal (Ed.), Behavior analysis of societies and cultural practices (pp. 39-73). Washington, DC: Hemisphere.

Glenn, S. S. (2004). Individual behavior, culture, and social change. The Behavior Analyst, 27 (2), 133-151.

Herrnstein, R. J. (1961). Relative and absolute strength of response as a function of frequency of reinforcement.
Journal of the Experimental Analysis of Behavior, 4, 267-272. doi: 10.1901/jeab.1961.4-267

Herrnstein, R. J. (1971). Quantitative hedonism. Journal Psychiatric Resesearch, 8(3), 399-412. doi: 10.1016/0022-3956(71)90033-1

Hunter, C. (2012). Analyzing Behavioral and Cultural Selection Contingencies. Revista Latinoamericana de Psicologia, 44, 43-54.

Marques, N. S., \& Tourinho, E. Z. (2015). The selection of cultural units by non-contingent cultural events. Behavior and Social Issues, 24, 126-140. doi:10.5210/bsi.v.22i0.4283

Morford, Z. H. \& Cihon, T. M. (2013). Developing an Experimental Analysis of Metacontingencies: Considerations Regarding Cooperation in a FourPerson Prisoner's Dilemma Game. Behavior and Social Issues, 22, 5-20. doi: 10.5210/bsi.v22i0.4207

Ortu, D., Becker, A. M., Woelz, T. A., \& Glenn, S. S. (2012). An Iterated Four-Player Prisoner's Dilemma Game with an External Selecting Agent: A Metacontingency Experiment. Revista Latinoamericana de Psicologia, 44, 111-120.

Pavanelli. S., Leite, F. L., \& Tourinho, E. Z. (2014). A "modelagem" de contingências comportamentais entrelaçadas complexas. Acta Comportamentalia, 22, 425-440.

Pierce, W. D. \& Cheney, C. D. (2013). Behavior Analysis and Learning (4th ed.). New York: Psychology Press.

Saconatto, A. T., \& Andery, M.A.P.A. (2013). Seleção por metacontingências: um análogo experimental de reforçamento negativo. [Selection by metcontingencies: An experimental analog of negative reinforcement.] Interação em Psicologia, 17, 1-10. doi: 10.5380/psi.v17i1.26779

Sampaio, A. A. S., Araújo, L. A. S., Gonçalo, M. E., Ferraz, J. C., Alves-Filho, A. P., Brito, I. S., Barros, N. M., \& Calado, J. I. F. (2013). Exploring the role of verbal behavior in a new experimental task for the study of metacontingencies. Behavior and Social Issues, 22, 87-101.

Schmitt, D. R., \& Marwell, G. (1968). Stimulus control in the experimental study of cooperation. Journal of the Experimental Analysis of Behavior, 11, 571-574. doi: 10.1901/jeab.1968.11-571

Sidman, M. (1953). Two temporal parameters of the maintenance of avoidance behavior by the white rat. Journal of Comparative and Physiological Psychology, 46, 253-261.

Sidman, M. (1960). Tactics of scientific research. New York: Basic Books.

Skinner, B. F. (1961). The Behavior of Organisms. New York: Appleton-Century-Crofts. (Publicação original: 1938).

Skinner, B.F. (1953). Science and human behavior. New York, NY: Free Press.

Smith, G. S., Houmanfar, R., \& Louis, S. J. (2011). The participatory role of verbal behavior in an elaborated account of metacontingency: From conceptualization to investigation. Behavior and Social Issues, 20, 122 146. 
Terrace, H. S. (1966). Stimulus control. In W. K. Honig (Ed.), Operant behavior: Áreas of research and application (pp. 271-344). New York: AppletonCentury-Crofs.

Todorov, J. C., \& Vasconcelos, I. (2015). Experimental analysis of the behavior of persons in groups: Selection of an aggregate product in a metacontingency. Behavior and Social Issues, 24, 111-125. doi: 10.5210/bsi.v24i0.5424

Toledo, T. F. N., Benvenuti, M. F. L., Sampaio, A. A. S., Marques, N. S., Cabral, P. A., Araújo, L. A. S., Machado, L. R., \& Moreira, L. R. Free culturant: a software for the experimental study of behavioral and Cultural Selection. Psychology \& Neuroscience, 8, 366-384.

Tourinho, E. Z., \& Vichi, C. (2012). Behavioral-analytic research of cultural selection and the complexity of cultural phenomena. Revista Latinoamericana de Psicologia, 44, 169-179.

Vichi, C. (2012). Efeitos da apresentação intermitente de consequências culturais sobre contingências comportamentais entrelaçadas e seus produtos agregados. Tese de doutorado, Programa de Pós Graduação em Teoria e Pesquisa do Comportamento, Universidade Federal do Pará, Belém.

Vichi, C., Andery, M. A. P. A., \& Glenn, S. S. (2009). A metacontingency experiment: the effects of contingent consequences on patterns of interlocking contingencies reinforcement. Behavioral and Social Issues, 18, 4157. doi: 10.5210/bsi.v18i1.2292

Zeiler, M. D. (1977). Schedules of reinforcement. In W. K. Honig \& J. E. R. Staddon (Eds.), Handbook of operant behavior (pp. 201-232). Englewood Cliffs, NJ: Prentice Hall. 